Open Access

\title{
Editorial
}

\section{ONCOGENIC HUMAN PAPILLOMAVIRUSES}

\section{High-Risk Human Papillomaviruses: Towards a Better Understanding of the Mechanisms of Viral Transformation, Latency and Immune-Escape}

\author{
A. Cid Arregui ${ }^{*}, 1$, , P. Gariglio ${ }^{2, \#}$, T. Kanda ${ }^{3, \#}$ and J. Doorbar ${ }^{4, \#}$ \\ ${ }^{I}$ Translational Immunology Department, German Cancer Research Center (DKFZ), Im Neuenheimer Feld 460, D- \\ 69120 Heidelberg, Germany \\ ${ }^{2}$ Department of Genetics and Molecular Biology, CINVESTAV-IPN, Mexico DF, Mexico \\ ${ }^{3}$ Center of Research Network for Infectious Diseases, Riken, Jimbocho 1-101, Chiyoda-ku, Tokyo 101-0051, Japan \\ ${ }^{4}$ Division of Virology, National Institute for Medical Research, London NW7 1AA, UK
}

High-risk human papillomaviruses (HR-HPVs) cause mucosal epithelial malignancies, most notably cervical intraepithelial neoplasia (CIN) and cancer of the uterine cervix. Prophylactic vaccines based on the L1 capsid proteins of the most prevalent HR-HPV types have been commercialized, and recent studies have shown that crossneutralization epitopes are present in the L2 minor capsid protein of HR-HPVs, thus giving hope for the prevention of many HPV-associated cancers. However, the high prevalence of HPV infections worldwide and the morbidity and mortality associated with them warrant further research to improve our understanding of HPV pathogenesis, and to develop new generations of prophylactic vaccines and effective immunotherapy strategies. Furthermore, there is a need for a better understanding of the virus life cycle and how this is influenced by the epithelial site, the mechanisms of viral persistence, regression and reactivation, and for detailed molecular studies that clarify the cellular pathways altered by the viral oncoproteins. This special issue was aimed to underline some of those aspects of current HPV research.

The E2 gene product of oncogenic HPVs is a transcriptional repressor that controls expression of the viral oncogenes. Integration of the viral DNA in the host genome resulting in disruption of the sequence encoding E2 was postulated more than two decades ago to be critical in the progression from premalignant lesions to cancer. However, further studies have shown that the viral DNA can be found in episomal as well as integrated state in HPV-associated cancers. Using E2-immunohistochemistry and NanoString technology to quantify E2 transcripts Xue et al. [1] show in their article that integration of the viral DNA in the host genome does not always cause disruption of the E2 gene and

\footnotetext{
*Address correspondence to this author at Translational Immunology, German Cancer Research Center DKFZ, Im Neuenheimer Feld 460, D69120 Heidelberg, Germany; Tel: +49 6221 567109; Fax: +49 6221 564776; E-mail: a.cid@dkfz.de

${ }^{\S}$ Guest Editor, ${ }^{\sharp} \mathrm{Co}$-Guest Editors
}

reduction of E2 transcripts, though expression of the E2 protein is dramatically reduced. While further studies should help unravel this paradox, this group proposes to use the relative amounts of E2 transcripts as a marker for progression to cancer.

E2 is an essential factor in the life cycle of all HPVs. In their review Muller and Demeret [2] suggest that the variability in the pathogenic potential of the different HPV types might result from differences in the interplay between viral and host proteins. A better understanding of proteinprotein interaction may lead to the development of new therapeutic strategies. They performed a DAVID (Database for Annotation, Visualization and Integrated Discovery) analysis of the known E2 interacting partners and found four main functional families targeted by E2: transcription factors, cell cycle, RNA processing and apoptosis. E2 has also partners in the ubiquitination and intracellular transport pathways. Such complexity suggests that E2 might drive different activities during the successive steps of the viral life cycle. Interestingly, Muller and Demeret could not identify any specific cellular targets with an impact on viral tropism or pathogenic capacity.

The question whether HPV infection may persist latent lifelong or is always a transient event has remained elusive to answer. This is due to the inherent limitations of HPV research: The lack of a convenient animal model for HRHPV infection and the fact that HPVs cannot be readily propagated in the laboratory. P. Gravitt [3] reviews the existing evidence for HPV latency and develops the model of cervical HPV latency and reactivation, which may account for many of the observations reported in the literature. These include the frequent detection of HPV in the absence of cervical disease, and the increased ability to detect cervical HPV in older women in some populations. Based on the study of latency in animal models, Maglennon and Doorbar [4] conclude that papillomavirus genomes can reside in a subset of epithelial cells following immune regression, with only a very low-level expression of viral gene products. In the Rabbit Oral Papillomavirus model, it appears that 
immune suppression can lead to a rise in viral copy number at regressed site, in agreement with our current thinking of latent reactivation in humans.

RNA interference (RNAi) is being developed as a powerful tool with high therapeutic potential. Yet, limitations of this technique, mostly related to RNAi delivery and biodistribution, have hindered its application. Singhania et al. [5] review current research on specific RNAi therapy for HPV, summarizing extensively all the RNAi studies carried out to date directed against HR-HPV types that have been undertaken in vitro and in vivo. Since viral transformation has been shown to require continuous expression of E6 and E7, these are the targets of choice for RNAi. However, knocking-down other viral genes like E2 and E5 seems also promising for therapeutic purposes. As mentioned above, a critical issue in the application of RNAi to therapy is to find an efficient delivery system. High-risk HPV types infect mucosae of the anogenital tract, the larynx and the oral cavity and, therefore, local application is possible thus overcoming the problems related to systemic delivery (degradation by nucleases, renal clearance, etc). Of the available methods, the PEGylated Lipoplex-entrapped Alginate Scaffold (PLAS) system developed by Singhania et $a l$, seems very promising in the vaginal application. In addition, there is the possibility of combining RNAi and Toll-like receptor (TLR) activities, which might result in stronger anti-viral and anti-tumor activity mediated by effectors of the innate immune system. Lastly, the search for new therapeutic targets may benefit from the use of synthetic siRNA libraries or shRNA to perform genome-wide RNAi screens in infected and non-infected cells, and thus identify so called lethality genes for cancer cells.

Genomic techniques have allowed large-scale analyses of gene expression in cervical cancers caused by HR-HPV and their derived cell lines, such as HeLa (positive for HPV18), and W12, SiHa and CaSki (positive for HPV16). The analysis of the transcriptomes of cells transformed by HPV compared to cells of normal cervical epithelium should improve our understanding of the mechanisms of HPV tumor promotion and progression. The transcriptome includes small non-coding micro-RNAs (miRNA), which can regulate the levels of specific mRNAs differentially in transformed and non-transformed cells. Kaczkowski et al. [6] review in detail the mRNA and miRNA global analyses of HPVpositive cell lines and tumors that have been reported to date. Microarray studies using RNA purified from the HPVpositive cell lines identified a number of genes that were either over- or under-expressed, such as interferon regulated genes (IFI44, MX1 and MX2) and cell cycle regulatory genes (MCMs, p16, TOPO2a). However, the variable pattern of gene expression obtained in the different studies, due to the diverse approaches and technologies that were employed, has made it difficult to reach a consensus. In cervical cancer tissues, mRNA profiling has shown over sixty up-regulated genes, most of them encoding transcription factors and proteins with relevant functions in apoptosis, DNA synthesis and repair, extracellular matrix, cytoskeleton and signal transduction. Interestingly, these studies have shown significant variability in the expression profile found in the different samples analyzed. This might reflect the distinct transforming capabilities of the HPV type(s) involved. Thus, for instance, HPV18 is the most prevalent in adenocarcinomas, while HPV16 causes most of squamouscell carcinomas. It may also reflect the fact that progression from HPV infection to cancer is a multistep event with cumulative cellular changes linked to the history of each patient: hygiene, food habits (vitamins A, C), other habits (smoking), contraceptives, number of sexual partners, etc.

It is generally accepted that, to be productive and persistent, HPV infection of the genital epithelia should take place in the basal cell layer. Microtrauma exposing the basal cells in the stratified epithelium of the ectocervix would be required. In the transformation zone, where the stratified squamous epithelium of the ectocervix meets the columnar epithelium of the endocervix, there are stem cells, which maintain both the columnar and the stratified epithelia continuous turnover. It is in this region where most cervical cancers arise and it is currently believed that infection of slow-cycling epithelial stem cells is necessary for infection to persist over a long period and eventually develop cancer. Nevertheless, it remains unclear whether HR-HPV types can directly transform a non-stem cell of the columnar epithelium or the basal layer of the stratified epithelium.

Assuming that HR-HPV types are able to infect stem cells in the transformation zone, it can be speculated that the viral genome remains in resting cells until it is activated and the expression of the viral oncogenes becomes deregulated and their encoded proteins drive cellular transformation. Indeed, some studies have shown a correlation between the expression of stem cell-associated genes, such as PSCA, PIWIL1, PIWIL2-like (PL2L), ALDH1 and TBX2, in squamous cell carcinoma biopsies. Further, cancer stem cells have been identified in spheroids isolated from cervical carcinoma tissues. These cells expressed stem-associated markers such as CD44, CK17, OCT4, PIWIL2, c-Myc, STAT3 and Sox2. Lopez et al. [7] review these and other aspects of our current understanding of the role of stem cells in the carcinogenesis induced by HR-HPV types in the cervical epithelium.

A main unsolved question related to HR-HPV infection is how these viruses are able to evade the immune defenses of an immune competent host over long periods of time. Although it has been estimated that $90 \%$ of new infections by HR-HPVs are cleared within one or two years, in $10 \%$ of cases infection persists and develops advanced CIN lesions and, eventually, cancer of the cervix. In these cases the host immune system fails to eliminate the infected cells and the virus. There are a number of viral and host factors that may contribute to regression. Concerning the virus, it has been shown that HR-HPV can promote local immunosuppression by various mechanisms: reducing the expression of HLA, turning down interferon-responsive genes, interfering with chemokines like CCL20, which could attract antigen presenting cells to the cervical epithelium, inducing attraction and retention of suppressive regulatory $\mathrm{T}$ cells or reducing the expression of toll-like receptors (TLRs). With regard to the host, there are individual factors known to increase the risk of developing CIN and cervical cancer, such as the use of hormonal contraceptives and smoking, as well as pathological conditions associated with reduced $\mathrm{CD} 4+\mathrm{T}$ cell functions, as it occurs in transplanted and HIV-infected patients. 
$\mathrm{T}$ cell responses but not antibody responses are required for regression of HR-HPV infection. Nevertheless, the precise immune mechanisms leading to regression or persistence of HPV infections are still incompletely understood. In particular, the immune responses to initial stages of HPV infection by innate effector cells, such as natural killer (NK) and NKT cells, remain obscure. The articles by Hibma and by Grabowska and Riemer [8, 9] review extensively these and other relevant aspects of HPV immunity.

With key questions on the interaction between HR-HPVs and the immune system still unanswered, it is not surprising that the development of efficient immunotherapy approaches to treat HPV-induced lesions has not succeeded yet. Indeed, none of the therapeutic vaccines or immunotherapies tested in clinical trials to date has convincingly cured HR-HPVinduced lesions or tumors. In his review, van der Burg [10] pinpoints the main pending questions in HR-HPV research and provides an in depth analysis of the $T$ cell responses found in HPV16-induced disease, underlying the relevance of specific CD4+ Th1 responses at earlier stages and of CD8+ $\mathrm{T}$ cell immunity in protecting against tumor progression, as well as the role that might play tumorassociated macrophages and the CCL2 chemokine produced by cervical cancer cells. Adoptive transfer of HPV-specific T cells to patients with HPV-induced cancer is to be reconsidered.

The ability of HPVs to escape the immune system by a variety of mechanisms obliges to the development of immunotherapy strategies that combine the activation of HPV-specific effector T cells, or their passive transfer, with methods that may enhance local immune reactivity, for instance by removing or neutralizing immune-suppressor cells or by attracting and stimulating antigen presenting cells. In their article, Kawana et al. [11] review previous clinical trials with therapeutic vaccines against HPV16induced CIN and cancer, and hypothesize that their limited success was due to the poor involvement of the cervical mucosal lesions in the immune responses evoked by the different vaccines tried. To improve the immune responses against HPV infection at cervical lesions they propose a vaccine based on Lactobacillus casei expressing the HPV16 E7 protein. Ongoing clinical trials involving HPV16-positive CIN3 patients immunized orally with this vaccine should prove their hypothesis. Juarez et al. [12] used in their studies a different approach based on virus-like particles (VLPs) of the hepatitis $\mathrm{B}$ surface antigen linked to cytotoxic $\mathrm{T}$ lymphocyte epitopes from HPV16 E7 and two immunostimulatory domains: CCL19 and IL-2. Immunization with such VLPs of HLA-A2 transgenic mice with humanized antigen presentation protected against tumor formation after challenge with a transformed cell line that constitutively expresses HPV16 E7.

Finally, another topic that deserves further consideration is the prevalence of the different HR-HPV types in cervical dysplastic lesions and cancer. An accurate knowledge of the HPV genotypes present in cervical lesions of a given population should help to formulate more adequate prophylactic vaccines covering such genotypes, and also to design therapeutic vaccines targeting the correct HPV types. An article by Kondo et al. [13] completes this special issue describing a study on the genotype prevalence of HR-HPVs in a cohort of Japanese women with abnormal cervical cytology. They used a reliable, low-cost genotyping method (PGMY-CHUV lineblot assay) described recently by the HPV Laboratory Network (LabNet) of the World Health Organization (WHO). They found that HPV16 was present in only one third of patients, which contrasts with the higher prevalence of this virus type in Western countries, while HPV52 and HPV58 are more prevalent. Interestingly, the study showed that about one third of the samples contained more than one genotype, a situation that is increasingly being observed also with the multiplex methods and which is of high relevance for the design of vaccines.

In summary, this special issue shows that in the HPV post-vaccine era our knowledge of these cancer-causing viruses is still very limited and deserves further efforts. The lessons learned with them will be very useful not only for a better understanding of the immunology of other cancerrelated viruses, such as the hepatitis $\mathrm{B}$ and $\mathrm{C}$ viruses, but also for the immunotherapy of non-viral cancers.

\section{REFERENCES}

[1] Xue Y, Lim D, Zhi L, He P, Abastado J-P, Thierry F. loss of hpv16 e2 protein expression without disruption of the e2 orf correlates with carcinogenic progression. Open Virol J 2012; 6: 163-72.

[2] Muller M, Demeret C. The HPV E2-Host Protein-Protein Interactions: a Complex Hijacking of the Cellular Network. Open Virol J 2012; 6: 173-89.

[3] Gravitt P. Evidence and Impact of Human Papillomavirus Latency. Open Virol J 2012; 6: 195-203.

[4] Maglennon GA, Doorbar J. The biology of Papillomavirus latency. Open Virol J 2012; 6: 190-7.

[5] Singhania R, Khairuddin N, Clarke D, McMillan NAJ. RNA interference for the treatment of papillomavirus disease. Open Virol J 2012; 6: 204-15.

[6] Kaczkowski B, Morevati M, Rossing M, Cilius F, Norrild B. A decade of global mRNA and miRNA profiling in HPV positive cell lines and clinical specimens. Open Virol J 2012; 6: 216-31.

[7] Lopez J, Ruíz G, Organista-Nava J, Gariglio P, García-Carrancá A. Human Papillomavirus infections and Cancer Stem Cells of tumors from the uterine cervix. Open Virol J 2012; 6: 232-40.

[8] Hibma MH. The immune response to papillomavirus during infection persistence and regression. Open Virol J 2012; 6: 241-8.

[9] Grabowska AK, Riemer AB. The Invisible Enemy - How Human Papillomaviruses Avoid Recognition and Clearance by the Host Immune System. Open Virol J 2012; 6: 249-56.

[10] van der Burg SH. Immunotherapy of Human Papilloma Virus induced disease. Open Virol J 2012; 6: 257-63.

[11] Kawana K, Adachi K, Kojima S, Kozuma S, Taketani Y. Human papillomavirus (HPV) therapeutic vaccines: a novel approach. Open Virol J 2012; 6: 264-9.

[12] Juarez V, Pasolli HA, Hellwig A, Garbi N, Cid Arregui A. Viruslike particles harboring CCL19, IL-2 and HPV16 E7 elicit protective T cell responses in HLA-A2 transgenic mice. Open Virol J 2012; 6: 270-6.

[13] Kondo K, Uenoyama A, Kitagawa R, Tsunoda H, KusumotoMatsuo R, Mori S, et al. Genotype Distribution of Human Papillomaviruses in Japanese Women with Abnormal Cervical Cytology Determined by PGMY-Lineblot Assay. Open Virol J 2012; 6: 277-83.

(C) Arregui et al.; Licensee Bentham Open.

This is an open access article licensed under the terms of the Creative Commons Attribution Non-Commercial License (http: //creativecommons.org/licenses/by$\mathrm{nc} / 3.0 /$ ) which permits unrestricted, non-commercial use, distribution and reproduction in any medium, provided the work is properly cited. 